\title{
The Relationship between Chemiluminescence Intensity and Genotoxicity in Polyaromatic Hydrocarbons and Aflatoxins
}

\author{
Kazumi Osada, ${ }^{1}$ Yuji Furukawa, ${ }^{1, *}$ Michio KomaI, ${ }^{1}$ \\ Kohya Hishinuma, ${ }^{2}$ Mika Kimura, ${ }^{2}$ Humio Inaba, ${ }^{2,3}$ \\ and Shuichi KIMURA ${ }^{1}$ \\ ${ }^{1}$ Laboratory of Nutrition, Faculty of Agriculture, Tohoku University, \\ Aoba-ku, Sendai 981, Japan \\ ${ }^{2}$ Biophoton Project, Research Development Corporation of Japan, \\ Aoba-ku, Sendai 980, Japan \\ ${ }^{3}$ Research Institute of Electrical Communication, \\ Tohoku University, Aoba-ku, Sendai 980, Japan
}

(Received June 4, 1994)

\begin{abstract}
Summary The chemiluminescence (CL) emitted from polyaromatic hydrocarbons (PAH) and aflatoxins (AF) in an $\mathrm{S} 9$ mix system can be detected by use of a chemiluminescence analyzer. These CL intensities were significantly correlated with the degree of mutagenicity as measured by the Ames test (using S. typhimurium TA100), and of clastogenicity measured by the micronucleus test using ICR mice. The emission spectra of the CL detected from aflatoxin B1 (AFB1) indicated a distinctive blue light around $440 \mathrm{~nm}$. In the case of AFs, there was a strong correlation between $\mathrm{CL}$ intensity and genotoxicity. CL intensity detected from nonsubstitutional polyaromatic hydrocarbons was significantly correlated with the degree of superdelocalizability. These CL emissions observed from PAHs and AFs not only correspond to the genotoxicity of these compounds but also reflect the electronic configuration of them.
\end{abstract}

Key Words: chemiluminescence, polyaromatic hydrocarbons, aflatoxins, micronucleus test, Ames test

There have been several reports about the chemiluminescence (CL) emission from carcinogens in a microsomal system or a post mitochondrial system ( $\mathrm{S} 9 \mathrm{mix}$ ). The first investigation into the emission of CL from carcinogens with a microsomal system was on benzo $(a)$ pyrene [1]. It was later shown that the typical

\footnotetext{
* To whom correspondence should be addressed.
} 
intermediate metabolite of benzo $(a)$ pyrene that is able to emit $\mathrm{CL}$ is 7,8-diol9,10-dioxetane $[2,3]$. Another study of $C L$ using a microsomal fraction system and concentrating on aflatoxin B1 (AFB1) and 9,10-dimethyl-1,2-benzanthracene (DMBA) was also reported [4].

Recently, using an S9 mix we tried to detect the CL from 11 different PAHs, 4 different AFs, and two kinds of nitroarenes. In these compounds we were able to detect strong $\mathrm{CL}$ from 5 different PAHs, namely, $\mathrm{B}(a) \mathrm{P}$, methylcholanthrene (MC), dibenzo( $a, i)$ pyrene (DBP), DMBA, and DBA $[5,6]$, and from 4 other compounds, AFB1, AFG1, 3-nitrofluoranthene, and dinitropyrene [7]. These 9 compounds are well known as strong carcinogens.

In the present study we investigated the relation between $C L$ intensity and the mutagenicity and clastogenicity of PAHs and AFs. We observed a significant correlation between CL intensity from those compounds and genotoxicity.

\section{MATERIALS AND METHODS}

Chemicals. Polyaromatic hydrocarbons used for this experiment (indicated in Table 1) were analytical grade and purchased from Wako chemical Industries Co., Osaka, or Sigma Chemical Co., St. Louis, MO. Phosphatidylcholine (type-IIIE), cholesterol, and aflatoxins came from Sigma Chemical Co. All organic solvents used in this study were analytical grade.

Preparation of $S 9$ mix. The $\mathrm{S} 9$ mix that was used in the chemiluminescence detection system was prepared by a slight modification of the method used by Yahagi [8]. Previous to homogenization, livers from decapitated male rats (100 g weight) were perfused through the portal vein with ice-cold $\mathrm{KCl}(150 \mathrm{~mm})$. The isolated livers were homogenized with 3 volumes of $\mathrm{KCl}(150 \mathrm{~mm})$ and centrifuged at $9,000 \times g$. To the resulting supernatant, NADPH was added at the concentration

Table 1. Comparison of the total CL intensity emitted from PAHs for $20 \mathrm{~min}$ and degree of genotoxicity measured by the Ames test (TA 100). Micronucleus test, and superdelocalizability $\left(\mathrm{IB}^{\prime}\right)$.

\begin{tabular}{lcccc}
\hline Compounds & $\begin{array}{c}\text { CL intensity } \\
(20 \mathrm{~min})\end{array}$ & $\begin{array}{c}\text { Revertants } \\
(\text { TA 100) }\end{array}$ & MNPCE & IB $^{\prime}$ \\
\hline Benzo $(a)$ pyrene & 151,870 & 801 & 13.8 & 2.358 \\
Dimethylbenzoanthracene & 63,300 & 509 & 21.0 & \\
3-Methylchoranthrene & 57,424 & 903 & 10.8 & \\
Dibenz $(a, h)$ anthracene & 24,781 & 491 & 4.6 & 2.318 \\
Pyrene & 10,661 & 179 & 3.25 & \\
Benzanthracene & 10,584 & 207 & 2.25 & 2.333 \\
Phenanthrene & 10,041 & 115 & 2.50 & 2.272 \\
Benzo(e)pyrene & 7,465 & 258 & & 2.301 \\
Triphenylene & 4,330 & 237 & 2.00 & 2.275 \\
Anthracene & 2,906 & 134 & 2.00 & 2.205 \\
\hline
\end{tabular}

The value of $\mathrm{IB}^{\prime}$ was from the results of Smith et al. [17]. The measurement procedure for $\mathrm{CL}$ and Ames test are given in Materials and Methods. 
of $4 \mu \mathrm{mol} / \mathrm{ml}$ as a co-factor. The mixture thus obtained was designated "S9 mix."

Preparation of lecithin-cholesterol vesicles. The lecithin-cholesterol vesicles were prepared by Batzri and Korn's method [9] and were used as the mutagen carrier in this monitoring system. Two hundred and twenty nmol of PAHs and 110 $\mathrm{nmol}$ of AFs were mixed with $900 \mathrm{nmol}$ of egg lecithin and $150 \mathrm{nmol}$ cholesterol and injected into $39 \mathrm{nmol}$ of sodium phosphate buffer $(5 \mathrm{ml})$. The compounds thus set in lecithin-cholesterol vesicles were added to the S9 mix system.

Measurement of ultra weak chemiluminescence. Chemiluminescence analyzer OX-71, a synchronous, single-photon counting apparatus manufactured by Tohoku Electronic Industries Co., Sendai, Japan, was used and equipped with a Hamamatsu R878 photomultiplier at the special response range of $300-650 \mathrm{~nm}$ (sensitivity maximum at $400 \mathrm{~nm}$ ). The sample solution, consisting of $2 \mathrm{ml}$ of $\mathrm{S} 9$ mix and $1 \mathrm{ml}$ of the polyaromatic hydrocarbon that was set in lecithin-cholesterol vesicles, was placed on a stainless-steel plate $(5.3 \mathrm{~cm}$ diameter, $1.3 \mathrm{~cm}$ height $)$, and monitored at $37^{\circ} \mathrm{C}$ for $20-30 \mathrm{~min}$. The emission intensity was expressed in terms of total counts for $20 \mathrm{~min}$. The spectral characteristics of the emission of AFB1 were measured by interposition of various Toshiba sharp-cut optical filters between the sample container and the photomultiplier.

Mutagenicity test. The mutagenic activity of compounds was tested by the standard method for mutagenicity assessment developed by Yahagi et al. [10]. Test bacteria, S. typhimurium TA100, together with a test compound, were incorporated into an agar overlay. In some experiments, the mixture of test compounds, S9 mixture, and bacteria were preincubated at $37^{\circ} \mathrm{C}$ for $20 \mathrm{~min}$, followed by the addition of soft agar [8]. Mutants (histidine-independent revertants) were enumerated after incubation at $37^{\circ} \mathrm{C}$ for $48 \mathrm{~h}$ in the dark. The test compounds were dissolved in dimethyl sulfoxide prior to usage. Three plates for solvent control and 4 plates for a test compound were included in each assay.

Micronucleus test. The experiment for PAHs was performed on ICR mice and that for AFs was performed on Sprague-Dawley rats procured from Funabashi Farm, Sendai, Japan. The animals used for this experiment were selected from those of similar age and weight. The compounds were used at a final concentration of 50 and $5 \mathrm{mg} / \mathrm{kg}$ body weight for PAHs and AFs, respectively, and the compounds were dissolved in corn oil before being injected into the animals. All treatments were administered by a single intraperitoneal injection, and the dose was adjusted to the weight of the animal.

The techniques used are described in greater detail elsewhere [11]. In brief, the mice were killed by cervical dislocation, and the femur was removed and stripped clean of muscle with gauze. A small opening was then made at both ends of the femur, and the marrow cavity was injected with $0.2 \mathrm{ml}$ of fetal calf serum (FCS) per femur to exude the bone marrow cells. After centrifugation at 1,000 rpm for $5 \mathrm{~min}$, the supernatant was discarded and the cellular pellet was resuspended in a small volume of the remaining FCS. The suspension was then placed and spread on a slide by use of the edge of a clean cover glass. The slides were kept at 
room temperature until the bone marrow cells had dried.

The slides were then fixed in absolute methanol for $1 \mathrm{~min}$; stained for $2 \mathrm{~min}$ in May-Gruenwald solution diluted with Sorensen buffer ( $\mathrm{pH}$ 6.8), 1:1; stained for $10 \mathrm{~min}$ in Giemsa diluted with Sorensen buffer, 1:40; rinsed in distilled water; dried clear in xylene for $5 \mathrm{~min}$; and finally mounted with a cover glass. One thousand polychromatic erythrocytes (PCE) were counted per animal, and the results were expressed as the average number of micronucleated PCE per 500 PCE.

Statistical analysis. Significant correlation coefficients between chemiluminescence intensity and the other parameters were obtained during the course of experiments by use of simple regression (significant level of $p<0.05$ ).

\section{RESULTS}

The total CL intensity, the number of revertant colonies of S. typhimurium TA100, and the number of micronucleated PCE (MNPCE) induced by the PAHs are shown in Table 1. The correlations between chemiluminescence intensity from polyaromatic hydrocarbons and mutagenicity measured by the Ames test (TA100) and clastogenicity measured by the micronucleus test are shown in Fig. 1 (a), (b).
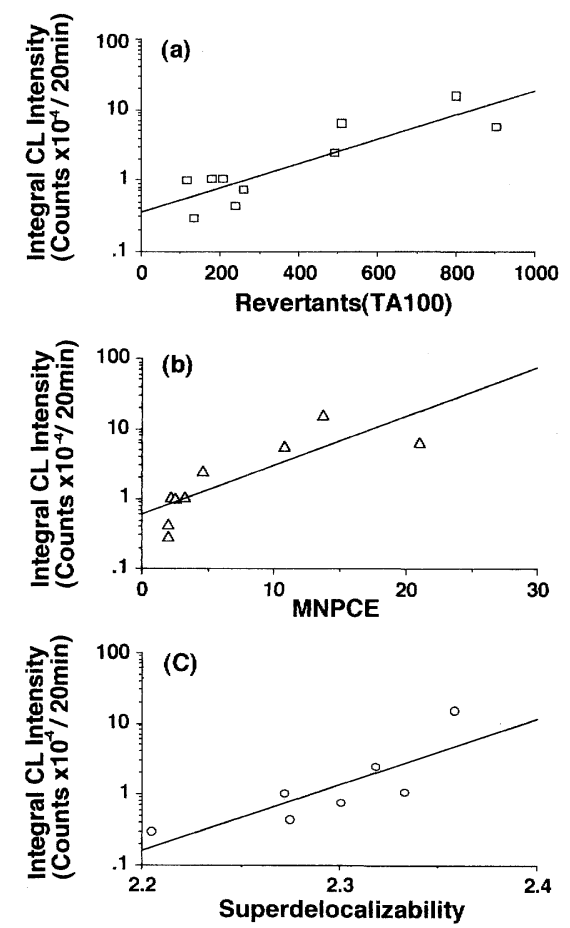

Fig. 1. The interrelationship between CL intensity and (a) Revertants, $y=(1.7096)^{-3} x+$ 3.5595, $r=0.873$; (b) MNPCE, $y=(6.9547)^{-3} x+3.7728, r=0.827$; and (c) index $\mathrm{IB}^{\prime}$, $y=9.2851 x-17.225, r=0.817$ measured from PAHs. 


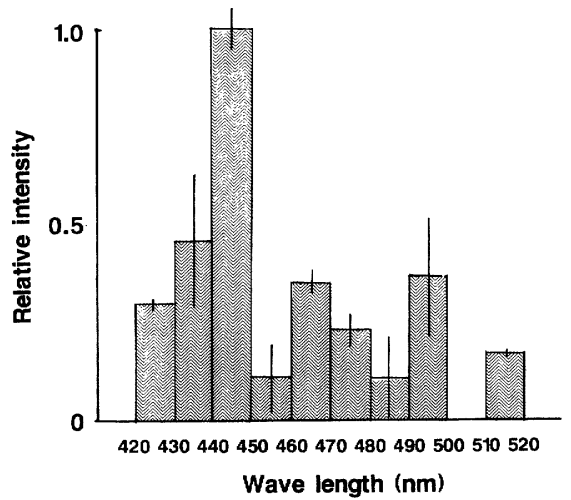

Fig. 2. Emission spectrum of chemiluminescence from aflatoxin $\mathrm{B} 1$ in the $\mathrm{S} 9$ mix system. The height of columns represents the tripricate mean of the relative CL intensity, and the vertical lines represent the SD. The highest peak is expressed as 1.0, and the others are expressed as proportional values.
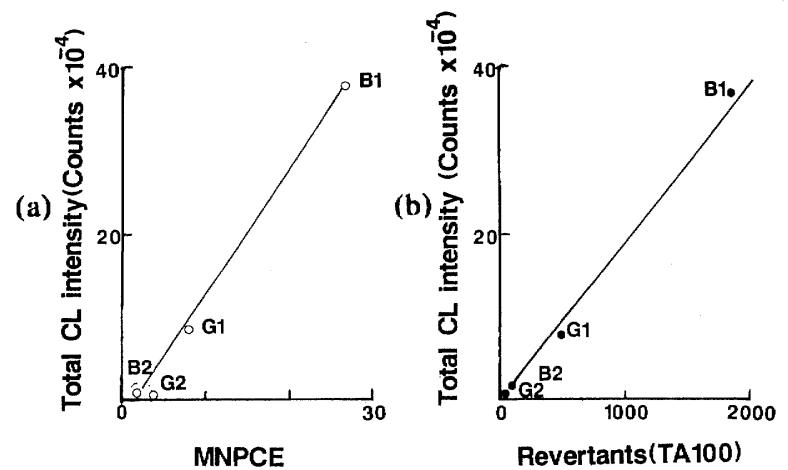

Fig. 3. Correlation between CL intensity and MNPCE (a) or Revertants (b) measured from aflatoxins. In both cases, $r=0.990$.

These CL intensities correlated significantly with the results of the tests. The correlation coefficients were $r=0.873$ and $r=0.827$, respectively.

There was a strong correlation $(r=0.817)$ between the superdelocalizability of PAHs and their CL intensity (Fig. 1(c)). In all three cases, CL intensities were plotted on a $\log$ scale.

The emission spectrum of CL detected from AFB1 is shown in Fig. 2. The special distribution of this light covered the wavelength range between $420-520$ $\mathrm{nm}$. The distinctive blue light peak at $440 \mathrm{~nm}$ was the major contributor to the total photoemission observed.

The correlations between chemiluminescence intensity from aflatoxins and degree of mutagenicity measured by the Ames test and of clastogenicity given by the micronucleus test are shown in Fig. 3. These CL intensities correlated strongly with the results of the tests. Both of the correlation coefficients were $r=0.990$. 


\section{DISCUSSION}

It was already known that there were several carcinogens that emitted CL in a post mitochondrial fraction system. This phenomenon was regarded as an uncommon observation, and restricted to special carcinogens, namely, $\mathrm{B}(a) \mathrm{P}$, $\mathrm{AFB} 1, \mathrm{MC}$, and so on, until recently.

In the present study, using an S9 mix system we could widely observe CL emissions from PAHs and AFs. The compounds that emitted significantly strong $\mathrm{CL}$ compared with the control were only carcinogens, namely, $\mathrm{B}(a) \mathrm{P}, \mathrm{MC}$, DMBA, DBA, AFB1, and AFG1. On the other hand, the weak or non carcinogenic compounds, namely, pyrene, benzanthracene, phenanthrene, benzo( $e$ )pyrene, triphenylene, anthracene, AFB2, and AFG2, didn't emit the CL significantly. In the case of both PAHs and AFs, there was a significant correlation between CL intensities from these compounds and degree of mutagenicity detected by the Ames test and of clastogenicity measured by the micronucleus test. These results suggest that CL emission from these compounds in an $\mathrm{S} 9 \mathrm{mix}$ system is closely related to the mechanisms responsible for the genotoxicity.

The Ames test was developed in 1971 as a simple mutagenicity test using a series of Salmonella typhimurium TA $[12,13]$. Nowadays this method is widely used as a primary screening method that is tried before experiments of in vivo carcinogenicity testing. From the result of the present study, our CL detection system may be applied as a novel mutagenicity detection system, at least for PAHs and AFs.

We observed a relationship between CL intensity and clastogenicity measured by the micronucleus test, too. This result indicates that $\mathrm{CL}$ intensities also agree with the findings of the in vivo assay system that detects the clastogenicity.

Sato et al. [14] demonstrated that with various PAHs containing quinones, there was a positive correlation between the mutation frequency in the Drosophila wing spot test and the CL intensity from Drosophila melanogaster fed those compounds. Our results do not contradict their data. It is worth noting that there is a positive correlation between $\mathrm{CL}$ intensity from PAHs and degree of carcinogenicity in two different species, namely, the rat and Drosophila.

On the other hand, we could only observe the significant correlation between $\mathrm{CL}$ intensity and genotoxicity in the compound categories of PAHs and AFs, suggesting that this correlation can only be observed with compounds that have a similar structure.

In the case of AFB1 the distinctive blue light around $440-450 \mathrm{~nm}$ was the major contributor to the total photoemission observed. This result absolutely differs to that of PAHs $[6,15]$. In the case of both $\mathrm{B}(a) \mathrm{P}$ and $\mathrm{MC}$, we have assigned their CL peak wavelength at around $500 \mathrm{~nm}[6]$. It was reported that 3-nitrofluoranthene, a kind of nitroarene, emitted red colored CL in an S9 mix system [16]. These observations suggest that a CL emission spectra may reflect the 
kind of compounds emitting CL.

Smith et al. [17] have reported that superdelocalizability of PAHs correlates strongly with carcinogenic potency. In the present study, we clarified that CL intensity also strongly correlates with this index. This result suggests that the mechanism of CL emission is related to the formation of an ultimate carcinogen and that CL intensity correlates with the carcinogenicity.

\section{REFERENCES}

1. Hamman, J.P., and Seliger, H.H. (1976): The chemical formation of excited states during hydroxylation of the carcinogenic hydrocarbon benzo $(a)$ pyrene by liver microsomes. Biochem. Biophys. Res. Commun., 70, 675-680.

2. Hamman, J.P., Seliger, H.H., and Posner, G.H. (1981): Specificity of chemiluminescence in the metabolism of benzo(a)pyrene to its carcinogenic diol epoxide. Proc. Natl. Acad. Sci. U.S.A., 78, 940-942.

3. Seliger, H.H., Tompson, A., Hamman, J.P., and Posner, G.H. (1982): Chemiluminescence of benzo(a)pyrene-7,8-diol. Photochem. Photobiol., 36, 359-365.

4. Emerole, G.O., and Dixon, R.L. (1980): Evaluation of chemiluminescence generation during microsomal metabolism of some carcinogens. Cancer Res., 40, 2002-2005.

5. Osada, K., and Kimura, S. (1988): Characteristics of chemiluminescence from mutagens in S9 mix. Mutat. Res., 203, 385 (abstract).

6. Osada, K., Furukawa, Y., Komai, M., and Kimura, S. (1990): Detection of ultra weak chemiluminescence from benzo $(a)$ pyrene and 3-methylcholanthrene. J. Clin. Biochem. Nutr., 8, 185-192.

7. Osada, K., Furukawa, Y., Komai, M., Kimura, M., Hishinuma, K., Saitoh, C., Inaba, H., and Kimura, S. (1991): Correlation between chemiluminescence intensity from mutagens and their mutagenicity. Mutat. Res., 252, 102-103 (abstract).

8. Yahagi, T. (1975): Screening methods using microbes for the environmental carcinogens. Protein, Nucleic acid, Enzyme, 20, 1178-1189 (in Japanese).

9. Batzri, S., and Korn, E.D. (1973): Single bilayer liposomes prepared without sonication. Biochim. Biophys. Acta, 298, 1015-1019.

10. Yahagi, T., Nagao, M., Seino, Y., Matsusima, T., Sugimura, T., and Okada, M. (1977): Mutagenicities of N-nitrosoamines on salmonella. Mutat. Res., 48, 121-130.

11. Schmid, W. (1975): The micronucleus test. Mutat. Res., 31, 9-15.

12. Ames, B.N., and Yanofsky, C. (1971): The detection of chemical mutagens with enteric bacteria, in Chemical Mutagens, Vol. 1, ed. by Hollaender, A., Plenum Press, New York, pp. 267-282.

13. Ames, B.N., Furton, W.F., Yamazaki, E., and Lee, G.D. (1973): Carcinogens are mutagens; A simple test system combining liver homogenates for activation and bacteria for detection. Proc. Natl. Acad. Sci. U.S.A., 70, 2281-2285.

14. Sato, T., Inaba, H., Kawai, K., Furukawa, H., Hirono, I., and Miyazawa, T. (1991): Low-level chemiluminescence from Drosophila melanogaster fed with chemical mutagens polycyclic hydrocarbon quinones and a carcinogenic bracken fern. Mutat. Res., 251, 91-97.

15. Hamman, J.P., Biggley, W.H., and Seliger, H.H. (1979): Emission spectrum of the microsomal chemiluminescence of a proximate carcinogen, 7,8-diol-benzo $(a)$ pyrene, determined with a wedge interference filter spectrometer. Biochem. Photobiol., 30, 519-524.

16. Osada, K., Furukawa, Y., Komai, M., Kimura, M., Hishinuma, K., Saitoh, C., Inaba, H., and Kimura, S. (1993): Novel detection of red-colored, ultra-weak chemiluminescence from carcinogens. J. Clin. Biochem. Nutr., 14, 1-6.

17. Smith, I.A., Berger, G.D., Seybold, P.G., and Serve, M.P. (1978): Relationships between carcinogenicity and theoretical reactivity indices in polycyclic aromatic hydrocarbons. Cancer Res., 38, 2968-2977. 